Classification

Physics Abstracts

$52.40 \mathrm{H}-62.50$

\title{
Simulation laser d'impacts de particules de très grande vitesse
}

\author{
J.-P. Bibring, F. Cottet $\left(^{*}\right)$, M. Hallouin $\left(^{*}\right)$, Y. Langevin et J.-P. Romain (*) \\ Laboratoire René-Bernas, 91406 Orsay, France \\ (*) E.N.S.M.A., 86034 Poitiers et GRECO I.L.M., Ecole Polytechnique, 91128 Palaiseau, France
}

(Reçu le 10 décembre 1982, accepté le 17 janvier 1983)

\begin{abstract}
Résumé. - Le laser au néodyme du GRECO I.L.M. délivrant des impulsions de l'ordre de $100 \mathrm{~J}$ en quelques ns, nous a permis de simuler des impacts de micrométéorites silicatées de quelques dixièmes de $\mu \mathrm{g}$, de vitesse comprise entre 5 et $45 \mathrm{~km} / \mathrm{s}$, sur une cible d'aluminium. Les cratères produits dans la cible sont hémisphériques, et le rapport $K_{\mathrm{m}}$, de la masse éjectée sur la masse de la particule incidente simulée, varie avec la vitesse d'impact $V_{\mathrm{p}}$ selon la loi $K_{\mathrm{m}}=-1,17 V_{\mathrm{p}}^{1,52}$.
\end{abstract}

\begin{abstract}
Silicated micrometeorites (mass : 1/10 $\mu \mathrm{g}$ ) impacting aluminium target are simulated with a neodyme pulsed laser, producing a maximum laser pulse energy of 100 in a few ns. The simulated micrometeorites velocities are in the range of $5 \mathrm{~km} / \mathrm{s}-45 \mathrm{~km} / \mathrm{s}$; the mass loss ratio, $K_{\mathrm{m}}$, is related to particle velocity $V_{\mathrm{p}}$ by $K_{\mathrm{m}}=1.17 V_{\mathrm{p}}^{1.52}$.
\end{abstract}

1. Introduction. - Les véhicules spatiaux subissent des impacts de micrométéorites dont la vitesse peut atteindre $80 \mathrm{~km} / \mathrm{s}$ et dont la masse se trouve essentiellement dans le domaine $10^{-5}$ à $10^{-10} \mathrm{~g}[1,2]$. C'est en particulier le cas des futures sondes qui vont réaliser un survol à moins de $20000 \mathrm{~km}$ de la comète de Halley, lors de son prochain passage au périhélie en 1986. Qu'il s'agisse de se protéger contre ces impacts ou de les utiliser pour en déduire certaines propriétés des grains cométaires, des simulations en laboratoire sont nécessaires. Les dispositifs expérimentaux actuels ne permettent pas d'atteindre des vitesses aussi grandes. Les grains de quelques micromètres sont accélérés au plus à une vingtaine de $\mathrm{km} / \mathrm{s}$. L'utilisation d'un laser pulsé de haute puissance pour simuler les impacts de grains, proposée par Pirri [3] et Nebolsine [4], pourrait permettre d'explorer le domaine des vitesses supérieures à $30 \mathrm{~km} / \mathrm{s}$. Nous avons effectué des tirs laser sur des cibles d'aluminium, à l'aide du laser à néodyme de haute énergie du GRECO I.L.M. à Palaiseau, afin de simuler l'impact d'une micrométéorite silicatée sur un blindage d'aluminium. Le domaine d'énergie accessible et la focalisation de ce laser nous ont permis d'atteindre des vitesses simulées de 5 à $45 \mathrm{~km} / \mathrm{s}$ pour des particules d'une masse de l'ordre du dixième de $\mu \mathrm{g}$.

2. Modèle de simulation par laser pulsé d'un impact de particule de grande vitesse. — Un impact laser, tout comme un impact de particule de grande vitesse, délivre à la surface de la cible une 
grande quantité de mouvement et une grande quantité d'énergie en un temps extrêmement court. Une onde de choc de grande amplitude (entre quelques centaines de kb et plusieurs $\mathrm{Mb}$ ) se propage alors dans la cible et s'y amortit progressivement. Selon la pression maximale atteinte localement, le matériau se volatilise, fond ou se fracture, ce qui provoque la formation d'un cratère autour du point d'impact.

Le principe de la simulation consiste à définir les caractéristiques d'une impulsion laser (durée $t$ de l'impulsion à mi-hauteur, énergie totale $E$, diamètre $D$ de la tache focale) induisant dans la cible une onde de choc similaire à celle produite par un projectile de diamètre $D_{\mathrm{p}}$, de longueur $L_{\mathrm{p}}$ (dans le cas d'un projectile cylindrique), de masse $M_{\mathrm{p}}$ et de vitesse $V_{\mathrm{p}}$ données. Si les deux processus créent des champs d'écoulement et de pression identiques, les déformations résultantes de la cible, et en particulier les dimensions du cratère, seront elles aussi identiques [5]. Les trois conditions à satisfaire sont les suivantes :

1) la pression initiale $P$ induite par l'impulsion laser doit être égale à celle engendrée par l'impact du projectile,

2) le diamètre $D$ de la tache focale doit être égal au diamètre $D_{\mathrm{p}}$ du projectile,

3) la durée $t$ de l'impulsion doit être telle que la quantité de mouvement transférée par l'impact laser à la cible soit égale à la quantité de mouvement du projectile :

$$
P t=\rho_{0} L_{\mathrm{p}} V_{\mathrm{p}},
$$

où $\rho_{0}$ est la densité initiale du projectile.

Pour une vitesse $V_{\mathrm{p}}$ donnée, la pression de choc $P$ induite à la surface de la cible se déduit des courbes de Hugoniot des matériaux constitutifs de la cible et du projectile. Dans le cas où ces matériaux sont identiques, le choc étant supposé monodimensionnel plan, la pression $P$ s'obtient à partir de la relation :

$$
P=\rho_{0}\left(a+b V_{\mathrm{p}} / 2\right) V_{\mathrm{p}} / 2
$$

où $a$ et $b$ sont les coefficients de la relation linéaire entre vitesse de choc $u$ et vitesse matérielle $u_{\mathrm{m}}$ :

$$
u=a+b u_{\mathrm{m}} .
$$

Dans le cas de l'aluminium, cette linéarité persiste jusqu'à des pressions très élevées [6], les coefficients $a$ et $b$ ayant pour valeur $5,386 \mathrm{~km} \cdot \mathrm{s}^{-1}$ et 1,339 respectivement. Nous considérerons que l'impact d'une micrométéorite silicatée sur une cible d'aluminium peut être assimilé à celui d'un projectile d'aluminium, les densités étant comparables, et les courbes de Hugoniot étant voisines à haute pression.

Dans le cas des tirs laser que nous avons effectués, une épaisseur de matière de l'ordre du $\mu \mathrm{m}$ est transformée en un plasma dense. La plus grande partie de l'énergie laser est transformée en énergie thermique du plasma. Celui-ci entre en expansion à grande vitesse, et soumet la cible à une pression $P$ approximativement constante pendant la durée de l'impulsion, et liée au flux incident du laser, $I$. Cette pression peut être déduite par une relation théorique $P(I)$, qui donne de bons résultats pour les flux compris entre $10^{12}$ et $10^{15} \mathrm{~W} / \mathrm{cm}^{2}[7]$ :

$$
P=5,5 \times 10^{-9}(\alpha I)^{2 / 3} .
$$

$P$ étant exprimé en $\mathrm{Mb}$, et $I$ en $\mathrm{W} / \mathrm{cm}^{2}$, et $\alpha$ représentant le taux d'absorption, qui dépend luimême de $I[8]:$ il varie de $\sim 80 \%$ pour $I=10^{12} \mathrm{~W} . \mathrm{cm}^{-2}$ à $\sim 50 \%$ pour $I=10^{14} \mathrm{~W} . \mathrm{cm}^{-2}$. Cette relation constitue vraisemblablement une limite supérieure, car elle ne tient pas compte des effets bidimensionnels, importants dans le cas d'impulsions « longues » (quelques ns) et très focalisées $(<100 \mu \mathrm{m})$.

Sur la base de ce modèle, les caractéristiques $\left(D_{\mathrm{p}}, L_{\mathrm{p}}, V_{\mathrm{p}}\right)$ du projectile dont l'impact est simulé 
par un tir laser de durée $t$, d'énergie totale $E$, et dont la tache focale a un diamètre $D$ sont évaluées de la manière suivante :

- le diamètre $D_{\mathrm{p}}$ du projectile est assimilé à $D$,

- pour déterminer la vitesse $V_{\mathrm{p}}$, nous calculons tout d'abord la pression $P$ à l'aide de la relation (4), le flux $I$ étant égal à $4 E /\left(\pi t D^{2}\right)$. On peut alors déduire $V_{\mathrm{p}}$ à partir de la relation (2), - $L_{\mathrm{p}}$ s'obtient alors aisément à partir de la relation (1).

$$
L_{\mathrm{p}}=100 \frac{P \times \tau}{\rho_{0} V_{\mathrm{p}}},
$$

$L_{\mathrm{p}}$ étant exprimée en micromètres.

La figure 1 donne le flux incident $I$ et la durée de l'impulsion laser $t$ en fonction de la vitesse $V_{\mathrm{p}}$ de la particule que l'on désire simuler, celle-ci ayant un diamètre $D_{\mathrm{p}}$ de $90 \mu \mathrm{m}$ et une longueur $L_{\mathrm{p}}$ de $90 \mu \mathrm{m}$ ou de $45 \mu \mathrm{m}$.

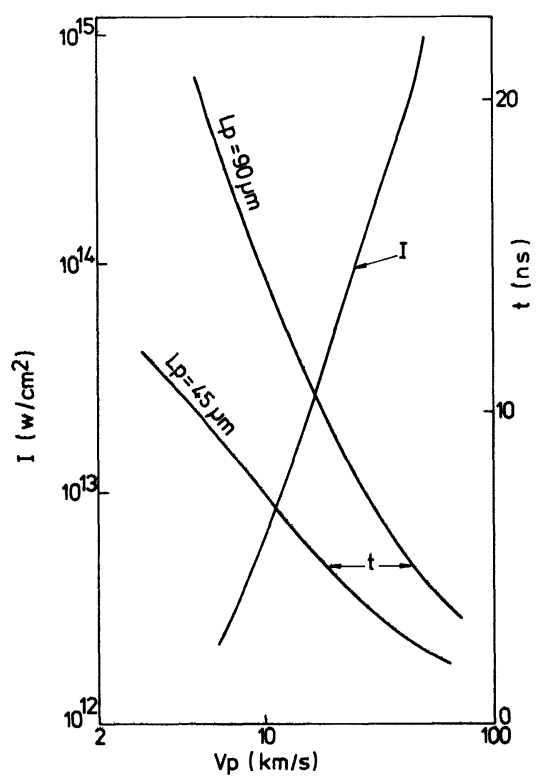

Fig. 1. - Flux incident $I$ et durée $t$ à mi-hauteur de l'impulsion laser en fonction de la vitesse $V_{\mathrm{p}}$ de la particule simulée, celle-ci ayant un diamètre de $90 \mu \mathrm{m}$ et une longueur de $90 \mu \mathrm{m}$ ou $45 \mu \mathrm{m}$.

[Laser requirements for hypervelocity impact simulation with a $90 \mu \mathrm{m}$ laser beam diameter.]

3. Résultats expérimentaux. - Le laser utilisé est le laser I.L.M. de l'école polytechnique. Il s'agit d'un laser au néodyme capable de délivrer des impulsions de plus de $100 \mathrm{~J}$ à une longueur d'onde de $1,06 \mu \mathrm{m}$. Le diamètre de la tache focale est déterminé en interposant une série de filtres de densité optique croissante, ce qui permet d'établir la répartition de l'énergie déposée dans le plan focal. La précision sur la mesure de $D$ est de $10 \%$. Ce diamètre était de $90 \mu \mathrm{m}$ pour une série de tirs d'énergie $60 \mathrm{~J}$ pendant $2,5 \mathrm{~ns}$, alors qu'il atteignait $110 \mu \mathrm{m}$ pour une série de tirs d'énergie $15 \mathrm{~J}$ pendant $4 \mathrm{~ns}$. Nous avons donc adopté ces deux valeurs de $D$, qui correspondent à la focalisation maximale, pour les tirs à " haute " et "basse " énergie respectivement. Nous avons également effectué quelques tirs défocalisés, le diamètre de la tache focale étant alors de $175 \mu \mathrm{m}$. 
Tableau I. - Résultats expérimentaux sur une cible épaisse en aluminium.

[Experimental results on thick aluminium target.]

\begin{tabular}{|c|c|c|c|c|c|c|c|c|c|c|}
\hline $\begin{array}{c}\text { Energie } \\
\mathbf{J}\end{array}$ & $\begin{array}{c}\text { Durée } t \\
\mathrm{~ns}\end{array}$ & $\begin{array}{c}D \\
\mu \mathrm{m}\end{array}$ & $\begin{array}{c}P_{\mathrm{c}} \\
\mu \mathrm{m}\end{array}$ & $\begin{array}{c}D_{\mathrm{c}} \\
\mu \mathrm{m}\end{array}$ & $\begin{array}{c}\text { Flux } I \\
10^{14} \mathrm{~W} / \mathrm{cm}^{2}\end{array}$ & $\begin{array}{c}P \\
\mathrm{Mb}\end{array}$ & $\begin{array}{c}V_{\mathrm{p}} \\
\mathrm{km} / \mathrm{s}\end{array}$ & $\begin{array}{c}L_{\mathrm{p}} \\
\mu \mathrm{m}\end{array}$ & $\begin{array}{c}M_{\mathrm{p}} \\
\mu \mathrm{g}\end{array}$ & $K_{\mathrm{m}}$ \\
\hline 90 & 2,3 & 90 & 350 & 775 & 6,137 & 22,08 & 45,43 & 41 & 0,711 & 420,2 \\
69 & 3,06 & 90 & 370 & 710 & 3,544 & 16,36 & 38,59 & 47 & 0,825 & 321,3 \\
64 & 2,5 & 90 & 340 & 660 & 4,024 & 17,59 & 40,16 & 40 & 0,697 & 302,2 \\
63 & 2,5 & 90 & 350 & 650 & 3,961 & 17,44 & 39,96 & 40 & 0,694 & 302,9 \\
61 & 3,06 & 90 & 360 & 670 & 3,134 & 15,22 & 37,09 & 46 & 0,799 & 287,7 \\
55 & 3,06 & 90 & 330 & 680 & 2,825 & 14,31 & 35,86 & 45 & 0,777 & 279,3 \\
47 & 3,06 & 90 & 330 & 625 & 2,414 & 13,01 & 34,02 & 43 & 0,744 & 246,2 \\
40 & 3,06 & 90 & 320 & 610 & 2,055 & 11,78 & 32,21 & 41 & 0,712 & 237,7 \\
87 & 2,3 & 175 & 427 & 745 & 1,573 & 9,97 & 29,34 & 29 & 1,879 & 179,3 \\
29 & 3,06 & 110 & 275 & 585 & 0,997 & 7,46 & 24,91 & 34 & 0,871 & 153,6 \\
29 & 4,13 & 110 & 310 & 605 & 0,739 & 6,62 & 23,27 & 43 & 1,117 & 144,4 \\
20 & 4,13 & 110 & 300 & 550 & 0,510 & 5,51 & 20,93 & 40 & 1,034 & 124,8 \\
16 & 4,13 & 110 & 280 & 500 & 0,408 & 4,88 & 19,49 & 38 & 0,982 & 101,3 \\
11 & 4,13 & 110 & 235 & 475 & 0,280 & 3,92 & 17,14 & 35 & 0,898 & 83,9 \\
28 & 21,8 & 175 & 390 & 720 & 0,054 & 1,43 & 9,15 & 125 & 8,193 & 35,1 \\
5 & 25 & 175 & 210 & 456 & 0,008 & 0,43 & 3,95 & 100 & 6,553 & 9,5 \\
\hline
\end{tabular}

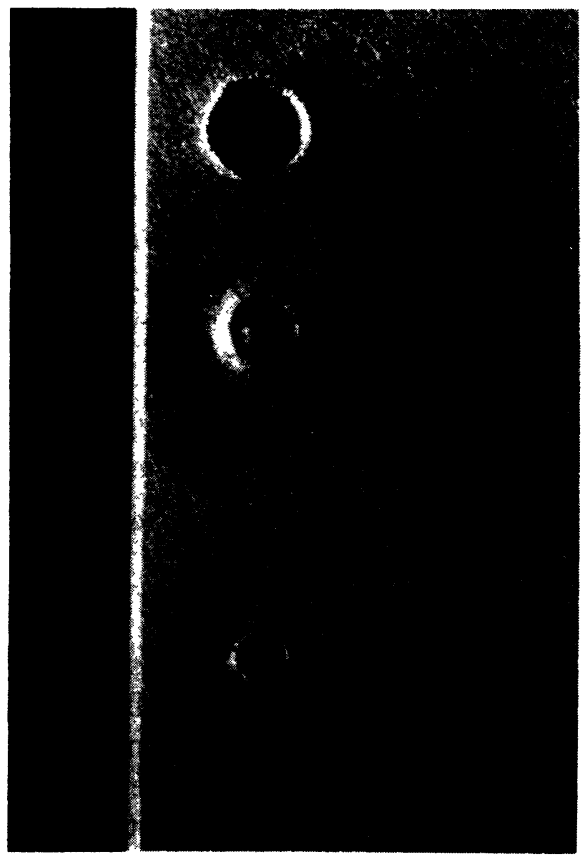

Fig. 2. - Microphotographies de cratères formés par des impacts laser d'énergie croissante dans une feuille d'aluminium de $1 \mathrm{~mm}$ d'épaisseur.

[Microphotography of craters produced by increasing power laser on an aluminium target (thickness $1 \mathrm{~mm})$.] 
Les cibles étaient constituées d'une feuille d'aluminium d'une épaisseur de $1 \mathrm{~mm}$.

Pour chaque impact laser, nous avons mesuré par microscopie optique le diamètre $D_{c}$ et la profondeur $P_{\mathrm{c}}$ du cratère formé, ce qui nous a permis d'évaluer la masse $M_{\mathrm{c}}$ expulsée du cratère. Pour chaque tir, nous avons déterminé les caractéristiques $D_{\mathrm{p}}, L_{\mathrm{p}}$, et donc la masse $M_{\mathrm{p}}$ du projectile simulé (supposé de densité 2,7) ainsi que sa vitesse $V_{\mathrm{p}}$.

L'ensemble des données obtenues est présenté sur le tableau I. On constate tout d'abord que les cratères formés sont quasi hémisphériques, dans tout le domaine d'énergie exploré. Cette forme correspond bien à celle prévue si l'onde de choc formée se dissipe dans un milieu homogène. En second lieu, ces cratères présentent le plus souvent des collerettes qui peuvent atteindre plusieurs dizaines de $\mu \mathrm{m}$ de haut (voir Fig. 2). La profondeur des cratères a été mesurée à partir du niveau de référence constitué par la face avant de la cible à l'extérieur de cette collerette.

Si l'on admet la validité du modèle que nous avons utilisé, ces tirs nous ont ainsi permis de simuler des impacts de particules d'une taille de $\sim 100 \mu \mathrm{m}$, jusqu'à des vitesses de $45 \mathrm{~km} / \mathrm{s}$. Ces caractéristiques sont très supérieures aux possibilités des meilleurs canons à particules actuels, et d'un ordre de grandeur proche des vitesses d'impact sur les engins spatiaux (jusqu'à $80 \mathrm{~km} / \mathrm{s}$ dans le cas de la sonde VEGA).

4. Discussion. - On admet en général qu'il y a invariance d'échelle dans le processus de formation d'un cratère dans un matériau homogène, c'est-à-dire qu'un projectile de même vitesse, et de dimensions doubles d'un autre projectile, produira un cratère dont les dimensions sont elles aussi doublées. Nous avons donc calculé le rapport $K_{\mathrm{m}}=M_{\mathrm{c}} / M_{\mathrm{p}}$, qui ne devrait dépendre que de la vitesse d'impact $V_{\mathrm{p}}$. La variation de $K_{\mathrm{m}}$ avec la vitesse simulée $V_{\mathrm{p}}$ est représentée sur la figure 3. Une approximation linéaire dans les coordonnées $\left(\log \left(K_{\mathrm{m}}\right), \log \left(V_{\mathrm{p}}\right)\right)$ permet d'obtenir la relation :

$$
K_{\mathrm{m}}=1,17 V_{\mathrm{p}}^{1,52} \quad\left(V_{\mathrm{p}} \text { en } \mathrm{km} / \mathrm{s}\right)
$$

On remarque que l'exposant de la vitesse est inférieur à 2 pour ces très grandes vitesses : la masse éjectée croît donc moins vite que l'énergie cinétique.

L'extrapolation à une vitesse d'impact de $80 \mathrm{~km} / \mathrm{s}$ donne les résultats suivants : une masse $M_{\mathrm{p}}$ de projectile devrait éjecter une masse 910 fois plus grande; pour une micrométéorite d'une taille de $100 \mu \mathrm{m}$, cela correspondrait à un cratère hémisphérique d'une profondeur de $610 \mu \mathrm{m}$.

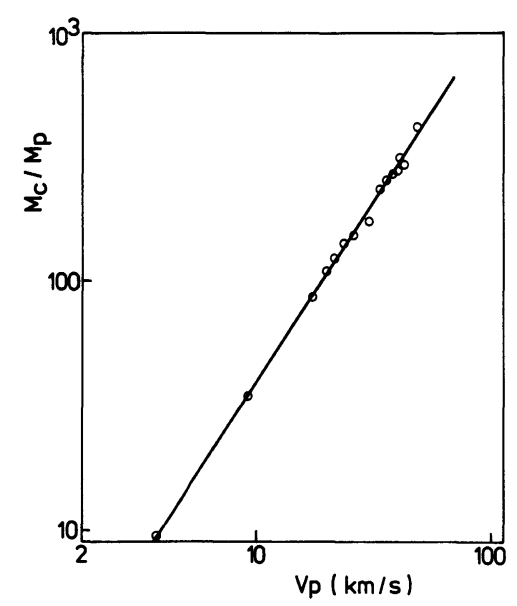

Fig. 3. - Variation de $K_{\mathrm{m}}$, rapport de la masse éjectée du cratère à la masse du projectile simulé, en fonction de la vitesse d'impact simulée.

[Mass loss ratio, $K_{\mathrm{m}}$, vs. simulated impact particle velocity.] 
La correspondance entre les cratères que nous avons observés et les caractéristiques des micrométéorites qui pourraient en former de semblables doit à présent être confirmée. En effet, elle repose sur un modèle simple, qui ne rend pas totalement compte de la complexité des processus liés à l'interaction laser-matière, et aux impacts à très grande vitesse. L'étape suivante va ainsi consister à calibrer les résultats correspondant au domaine des faibles vitesses $(10$ à $20 \mathrm{~km} / \mathrm{s})$, accessible avec les canons à poussière existants, tel celui du Max Planck Institüt à Heidelberg. On pourrait alors préciser le coefficient constant de la relation (6) qui relie la masse éjectée à la vitesse de la particule incidente.

Remerciements. - Nous tenons à remercier E. Fabre et toute l'équipe du laser du GRECO I.L.M. qui ont rendu ces expériences possibles, ainsi que R. Pellat pour ses encouragements et ses conseils.

\section{Bibliographie}

[1] Eichelberger, P. J., Gehring, J. W., Am. Rocket Soc. 32 (1962) 1583.

[2] Burton, W. M., XXIV COSPAR Symposium No 6 Impact Processes of solid Bodies, Ottawa (1982).

[3] PIrri, A. N., Phys. Fluids 20 (1977) 221.

[4] Nebolsine, P. E., AIAA 14th Aerospace Sciences Meeting, Washington, D.C. (1976).

[5] Riney, T. D., in High Velocity Impact Phenomena, R. Kinslow éditeur (Academic Press, New York) 1970 , p. 157.

[6] Mitchell, A. C. and Wellis, W. J., J. Appl. Phys. 52 (1981).

[7] FabBro, R., Thèse de l'Université Paris XI (1982).

[8] Garban-Labaume, C., Fabre, E., Max, C. E., Fabbro, R., Amiranoff, F., Virmont, J., Weinfeld, M. and Michard, A., Phys. Rev. Lett. 48 (1982) 1018. 